\title{
Use of Pentoxypline and Tocopherol in the treatment of Osteorradionecrosis: a literature review
}

\author{
Jonas Nogueira Ferreira Maciel Gusmão 1*, George Matos Ferreira Gomes Júnior 1, Roberto Dias Rêgo 1, \\ Eliardo Silveira Santos 1 \\ ${ }^{1}$ Department of Oral and Maxillofacial Surgery, Hospital Geral de Fortaleza, HGF, Fortaleza, Brazil. \\ *Corresponding author: Jonas Nogueira Ferreira Maciel Gusmão. Ávila Goularte, $n^{\circ} 900$-Papicu. Zip Code: 60150- \\ 160 -Ceará, CE, Brazil. Phone: +55 (85)9 8141-6401. E-mail: jonasnferreira@yahoo.com.br
}

Received on: May 15, 2021. Accepted on: May 30, 2021. Available online: Jun 4, 2021.

\begin{abstract}
Osteoradionecrosis (ORN) is a complication of radiotherapy (RT), which affects patients with head and neck cancer. Once established, an ORN does not disappear spontaneously and a standard treatment has not yet been defined. Treatment is often complex and multimodal. With a better understanding of the pathophysiology of ORN, new treatments and possibilities for the most predictable results of this difficult prognosis pathology arise. Under these circumstances, good results have been observed with an association of peripheral vasodilator drugs and antioxidants, such as pentoxifylline and tocopherol. The objective of this work was, through a review of the literature, to describe a pathophysiology of the ORN of the jaws and discuss as new perspectives of a conservative treatment with pentoxifylline and tocopherol. It was observed as an alternative to the drug association as a therapeutic alternative of the ORN, mainly in the early stages, it showed good results; However, it requires more controlled clinical studies to measure and consolidate the benefits that this treatment can provide patients.
\end{abstract}

Keywords: Pentoxifylline; Tocopherol; Osteoradionecrosis.

\section{Introduction}

Head and neck cancer worldwide represents $10 \%$ of malignant tumors. It is estimated, for Brazil, in 2016, 11,140 new cases of cancer of the oral cavity in men and 4,350 in women. These values correspond to an estimated risk of 11 new cases per 100,000 men and 4 per 100,000 women [1].

The recommended treatment for these neoplasms is through surgery, Radiotherapy (RT) and Chemotherapy (QT), with only one method or the association of two or more methods, depending on the characteristics of the disease. However, despite the good results obtained with RT, complications arose from its use, resulting in reduced quality of life, interference in therapy and increased morbidity [2-3].

The main complications of using RT are mucositis, xerostomia, candidiasis, dysgeusia, caries, soft tissue necrosis and osteoradionecrosis (ORN). Despite better control of risk factors seen today, such as greater attention to 
oral hygiene and dental health care, and modern advances in RT methods, ORN remains a reality today [5].

RT has been used in the treatment of malignant tumors of the head and neck region, exclusively, or associated with surgery and chemotherapy. It is known that radiosensitization of cells adjacent to the tumor can limit the treatment and, depending on the irradiated field, result in important morbid sequelae [3].

Due to medical advances, patients undergoing radiotherapy or chemotherapy, even those with acute or chronic side effects, have achieved a longer survival. This has resulted in an increase in the demand of such patients seeking dental treatment, making it essential that the Dental Surgeon is aware of such effects, as well as their relationship with dental treatments [5].

The need to maintain the oral health of cancer patients undergoing RT aims to provide a better quality of life. The periodontium must be maintained in healthy condition through procedures performed routinely, before and during irradiation. Guidance on oral hygiene techniques, motivation and the patient's ability to cooperate are essential to achieve the best prognosis [2].

ORN is characterized as one of the late adverse effects of RT [5]. For Marx [6], ORN can be considered as an ischemic necrosis, with superficial infection of the exposed bone characterized by hypocellularity, hypoxia, hypovascularization, with the mandible being the structure that presents a greater predisposition to the development of osteonecrosis, not only because of its dense bone configuration as well as the type of blood supply [5, 7].

The treatment of ORN is still a challenge for the clinician. Currently, it seems to be a consensus that the ORN should be handled, initially in a conservative way, through debridement and cleaning of surgical wounds with antimicrobial solutions, antibiotic therapy and surgical debridement [8].

Conservative treatment includes local irrigation (with saline solutions, chlorhexidine, hydrogen peroxide or povidone iodine), curettage of the lesion, elimination of small sequestrations, with or without the use of hyperbaric oxygenation. The elimination of habits, such as tobacco and alcohol, elimination of traumatic prostheses, use of hygiene measures and antiseptic mouthwashes several times a day, use of analgesics, are part of conservative therapy. Other therapeutic approaches include Hyperbaric Oxygen Therapy (HBO), use of peripheral vasodilator drugs and antioxidants, laser therapy, platelet aggregates, ozone therapy and ultrasound [9-10].

The use of peripheral vasodilator drugs and antioxidants, such as pentoxifylline and tocopherol, in the treatment of patients with maxillary ORN, has shown promising results, which may or may not be associated 
with Clodronate, in cases of progressive ORN to obtain maximum benefit [11].

In this context, the present study aimed, through a literature review, to describe the pathophysiology of maxillary ORN and to discuss the new perspectives of a conservative treatment with pentoxifylline and tocopherol.

\section{Methodology}

A literature search was performed by consulting the following databases: ScienceDirect, MedLine, and Scielo. The keywords used were selected by listing the following combinations: "Pentoxifylline", "Tocopherol", "Osteoradionecrosis", "Jaw", "Pentoxifylline", "Tocopherol", "Osteoradionecrosis". The inclusion criteria used in this bibliographic search were: full text, written in Portuguese and English. The articles used are casecontrol studies, retrospective studies, bibliographic reviews and systematic reviews with up to 15 years of publication. From the totality of articles found, all those whose theme and objectives were not related were excluded. articles on osteoradionecrosis that were not related to the oral cavity, maxilla and mandible were also excluded.

\section{Results (Review)}

\section{Head and Neck Cancer}

Cancer is the name given to a set of more than 100 diseases that have in common the disordered growth of cells that invade tissues and organs and can spread to other regions of the body. This uncontrollable reproduction of cells tends to be very aggressive, determining the formation of tumors or malignant neoplasms [1].

Based on the document World Cancer Report, 2014, by the International Agency for Research on Cancer (IARC), of the World Health Organization (WHO), it is unquestionable that cancer is a public health problem, especially among developing countries, where it is expected that, in the coming decades, the impact of cancer on the population will correspond to $80 \%$ of the more than 20 million new cases estimated for 2025 [1].

The National Cancer Institute (INCA) revealed that cancer of the oral cavity has an incidence in the population around $40 \%$ of all malignant tumors that affect the head and neck region. Approximately 300 thousand new cases were estimated in the world in 2012, and of these, approximately two thirds are in males. For mortality, 145 thousand deaths from cancer were estimated in the world in 2012 [1].

Without considering nonmelanoma skin tumors, cancer of the oral cavity in men is the fourth most frequent in the Southeast (14.58/100 thousand). In the Northeast (6.86/100 thousand) and Midwest regions, it occupies the fifth position (9.15/100 thousand). In the South Region (15.91/100 thousand), it occupies the 
sixth position, and in the North Region (3.46/100 thousand) it is the seventh most frequent. For women, it is the ninth most frequent in the Northeast Region (4.11/100 thousand). In the Southeast Region (5.29/100 thousand), it occupies the tenth position. In the North $(1.76 / 100$ thousand) and Midwest (2.79/100 thousand), it is the 12 th most frequent, and in the South (3.32/100 thousand), it occupies the 15th position [1].

With the gradual evolution in the treatment of carcinomas in the head and neck region, the cure rate has grown substantially. The patient can be treated with surgery, RT and chemotherapy concomitantly or not [3]. Combined surgery with RT has been used in the treatment of advanced malignant tumors of the head and neck, providing higher cure and survival rates than the isolated treatment [12].

Complications resulting from radiotherapy treatment depend, in addition to the total dose, on other factors, such as: type and radiosensitivity of healthy irradiated tissue, dose fractionation, age, systemic conditions, some addictions such as alcoholism and smoking, and inadequate oral hygiene [7].

High doses of radiation can cause hypoxia, reduced blood supply, necrosis and, consequently, infection. Likewise, the skin and mucosa found in the irradiated field can undergo changes such as desquamation, blistering, erythema, necrosis, as well as pain and burning in the most severe cases, favoring the development of structural and functional tissue changes [13].

Vissink et al. [14], in their studies, evaluated the forms of treatment and the prognosis of patients with squamous cell carcinoma of the oral cavity. They stated that a cure for this carcinoma can be achieved with surgery, RT and a combination of both. They also considered the problem that oral cavity tumors are still not adequately treated, emphasizing the need for diagnosis in the early stages.

A protocol that minimizes the sequelae of RT must be previously instituted and monitored before, during and after the end of the treatment. A comprehensive multidisciplinary clinical approach allows preventing, diagnosing and controlling the side effects of RT. Psychological aspects are relevant and the patient's self-esteem can favor adherence to the proposed treatment, personal care and with their teeth [2].

\section{Radiotherapy and its consequences}

RT is a treatment modality for malignant tumors, such as squamous cell carcinoma, whose therapeutic agent is ionizing radiation. This radiation is capable of creating unstable atoms that damage the cell's DNA and prevent the neoplastic cell from replicating. The radiation dose is measured in gray units (Gy), and generally, patients with head and neck carcinomas receive, as a 
curative dose, between 50 and 70 Gy $(1$ $\mathrm{Gy}=1 \mathrm{~J} / \mathrm{kg}=100 \mathrm{rads})$. This dose is usually applied in fractions, for a period of five to seven weeks, once a day, five days a week and with a daily dose in the tumor around $2 \mathrm{~Gy}$. In doses low of 10 Gy there is already the appearance of side effects (radiation skin syndrome, mucositis and glandular alterations) [14].

Ionizing radiation is divided into corpuscular and electromagnetic radiation. The corpuscular ones are represented by electrons, protons and neutrons; the electromagnetic ones are called photons, being represented by $\mathrm{X}$ rays and gamma rays. They act on nuclear DNA leading to death or loss of their reproductive capacity. However, the ability to multiply varies according to cell type. Thus, there is a radiosensitivity scale for both tumor cells and normal cells. Embryonic neoplasms and lymphomas are radiosensitive tumors, while carcinomas are moderately radiosensitive [15].

However, RT has been associated with many deleterious effects such as mucositis, xerostomia, radiation caries, dysgeusia, radiodermatitis, fibrosis and ORN. The occurrence of these reactions depends on the radiation dose, the location of the irradiated area, age and systemic conditions and other concomitant treatments [16].

As for the treatment of head and neck CA, ORN is a sequelae arising from RT, characterized by the loss of skin tissue and/or mouth lining mucosa that progresses to the consequent exposure of necrotic bone tissue after a minimal period, in general from three to six months, but which can appear after treatment indefinitely [14].

$\mathrm{RN}$ is one of the most severe complications of RT, occurring seven times more in the mandible than in the maxilla, due to its high bone density and less vascularization. About $74 \%$ of cases occur within the first three years after RT. Ionizing radiation makes the vascular channels narrow, which reduces blood flow, producing an area that is poorly resistant to trauma and poorly regenerated. Therefore, traumatic procedures that can promote bone necrosis are not indicated. Extractions, for example, must be performed at least two weeks before radiotherapy treatment [17].

Predisposing factors commonly related to ORN of the mandible include poor oral hygiene, periodontal disease, dentoalveolar abscess, extensive caries, anatomical site of the tumor, increasing doses of RT, and dentoalveolar surgery during RT or in the postoperative period [2].

In 1983, Marx [6] described the first pathophysiological theory about ORN, which has been the most accepted since then. According to the author, radiation would cause tissue changes resulting in hypoxia, hypocellularity and hypovascularization, which 
promotes a disturbance in tissue healing.

Nabil and Samman [4] critically reviewed 19 selected articles to analyze the incidence and factors influencing ORN development after tooth extraction in irradiated patients. They found a total incidence of $7 \%$ of ORN after tooth extraction in irradiated patients. When extractions were performed in conjunction with prophylactic HBOT, the incidence of ORN was $4 \%$, whereas when extraction was preceded by prophylactic antibiotics, the incidence was 6\% [4].

The authors concluded that, although the incidence of ORN after post-irradiation tooth extractions was low, the extraction of mandibular teeth within the radiation field, in patients who received doses greater than $60 \mathrm{~Gy}$, represents a greater risk for the development of ORN [4].

ORN is a complex and multifactorial process with vascular alterations (endothelial, perivascular, and microvascular tissue) and cellular components (both intracellular and extracellular), induced by ionizing radiation. It also causes dysregulation of fibroblastic activity, alters the expression of inflammatory cytokines and growth factors, leading to fibrosis, disturbance of normal bone remodeling and cell death. Therefore, local trauma can act as a reinforcing factor for its onset; scheme described in (Figure 1) [18].
Several classifications have already been proposed for the ORN. Tables 1 to 3 show the classifications proposed by Notani et al. [19], Støre et al. [20] and Epstein et al. [21], respectively. There is still no consensus on the most appropriate classification for the disease [13].

\section{New treatment options for osteoradionecrosis}

The management of ORN is difficult and not always successful because of the lack of and effective prevention methods, accompanied by several risk factors. Maxillary ORN is usually treated conservatively or surgically. Conservative therapies include frequent irrigation with saline, 0.12\% chlorhexidine solutions, povidone-iodine solutions, and antibiotic therapy during periods of active infection. Another conservative approach is the use of HBOT [22].

HBOT would increase oxygen supply to tissues in hypoxia, which would stimulate fibroblast proliferation, collagen formation and angiogenesis.

Its use has been advocated in the pre-and postoperative period of patients at high risk of tooth extraction or surgical intervention. In addition, it can also be used in small lesions, together with other conservative measures, in order to avoid extensive surgical resections of the mandible, obtaining an $81 \%$ response rate of osteoradionecrosis to treatment with HBOT, associated 
with a reduction in symptoms of patients [23].

Figure 1. Representative scheme of the pathophysiological mechanisms involved in osteoradionecrosis (ORN): Fibroatrophic theories. TNF- $\alpha$, Tumor Necrosis Factor-a; PDGF, Platelet-derived growth factor; IL, interleukin; FGF, Fibroblast Growth Factor; TGF- $\beta 1$, Tumor growth factor- $\beta 1$.

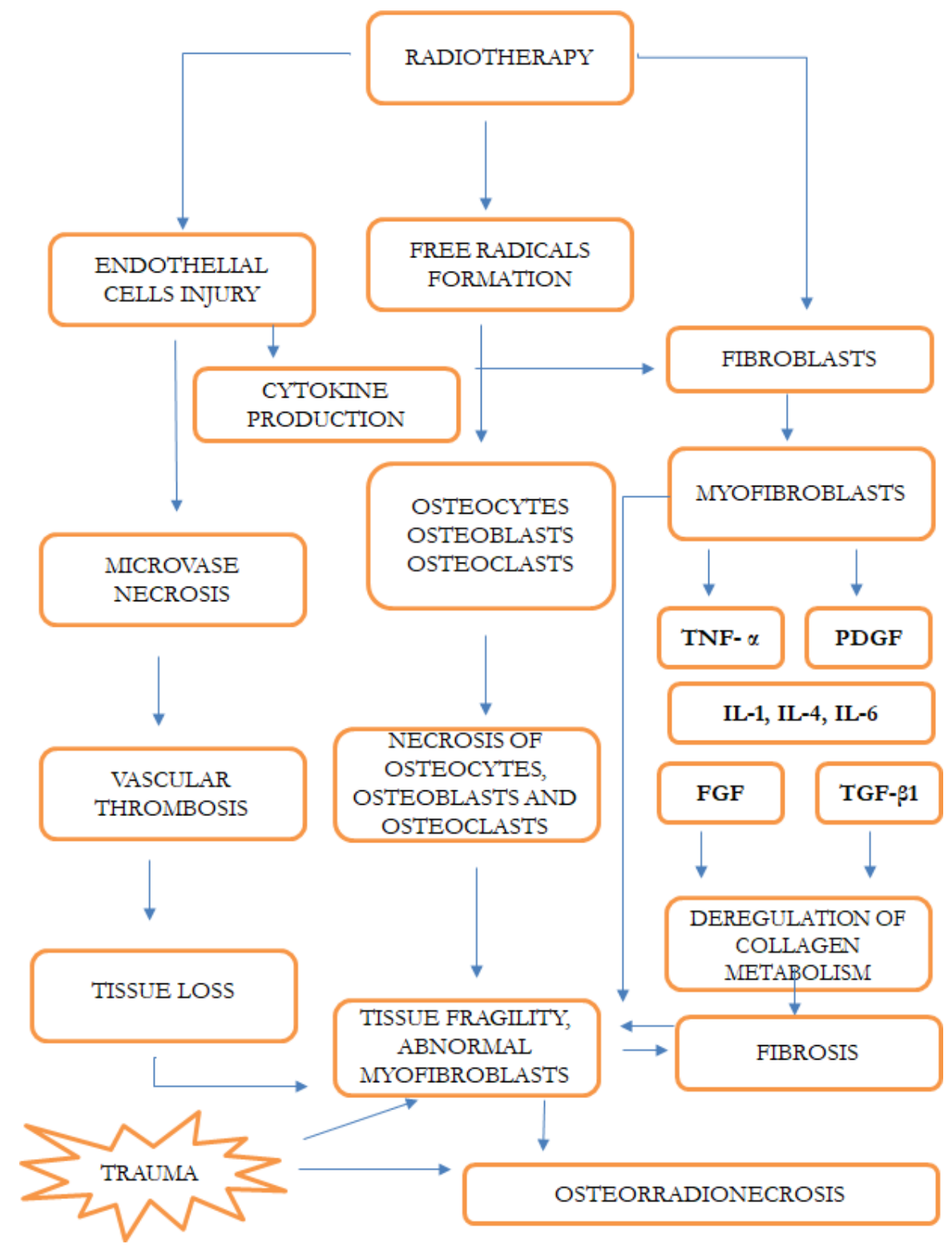

Adapted from Lyons and Ghazali (2008). 
Table 1. Classification of osteoradionecrosis, according to Notani et al. [19].

\begin{tabular}{ll}
\hline Staging & Description \\
\hline Grade I & Limited to alveolar bone \\
\hline Grade II & Up to the inferior dental canal \\
\hline Grade III & Exceeding the dental canal, or with pathological fracture or fistula \\
\hline Adapted from Notani et al. [19]. \\
Tabela 2. Classificação da osteorradionecrose segundo Støre et al. [20]. \\
\hline Staging & Only mucosal defect \\
\hline 0 & Radiological evidence of necrotic bone, but with intact mucosa \\
\hline I & Radiological osteonecrosis with bone denuded intraorally \\
\hline II
\end{tabular}

Adapted from Støre et al. [20].

Tabela 3. Classificação da osteorradionecrose segundo Epstein et al. [21].

\begin{tabular}{ll}
\hline Staging & \multicolumn{1}{c}{ Description } \\
\hline I & $\begin{array}{l}\text { Resolved, Healed } \\
\text { (A) Absence of fracture } \\
\text { (B) Pathological fracture }\end{array}$ \\
\hline II & $\begin{array}{l}\text { Chronic, persistent } \\
\text { (A) Absence of fracture } \\
\text { (B) Pathological fracture }\end{array}$ \\
\hline III & Active, Progressive \\
& (A) Absence of fracture \\
& (B) Pathological fracture \\
\hline
\end{tabular}

Adapted from Epstein et al. [21].

Marx et al. [6] compared HBOT to penicillin in the prevention of osteoradionecrosis after tooth extraction, showing that the group that used only HBOT showed an incidence of the disease of $5 \%$, while in the group that used only penicillin the rate was $29.9 \%$.

Treatment options are selected according to the stage of the ORN; PHASE I, or early stage ORN - is usually 
treated conservatively with local wound care, HBOT, and antibiotic therapy when necessary; PHASE II, intermediate stage of ORN - stage of difficult definition of a standard treatment; PHASE III, advanced stage ORN - is usually surgically treated with wide resection and immediate microvascular reconstruction [17].
Recent advances in understanding ORN pathogenesis have resulted in a new therapeutic strategy designed to improve tissue healing with a combination of pentoxifylline and tocopherol. Fan et al. [17] suggested that treatment options for ORN of the mandible could combine the three main approaches already cited for ORN control (Figure 2).

Figure 2. Recent treatment options for managing osteoradionecrosis of the jaw.

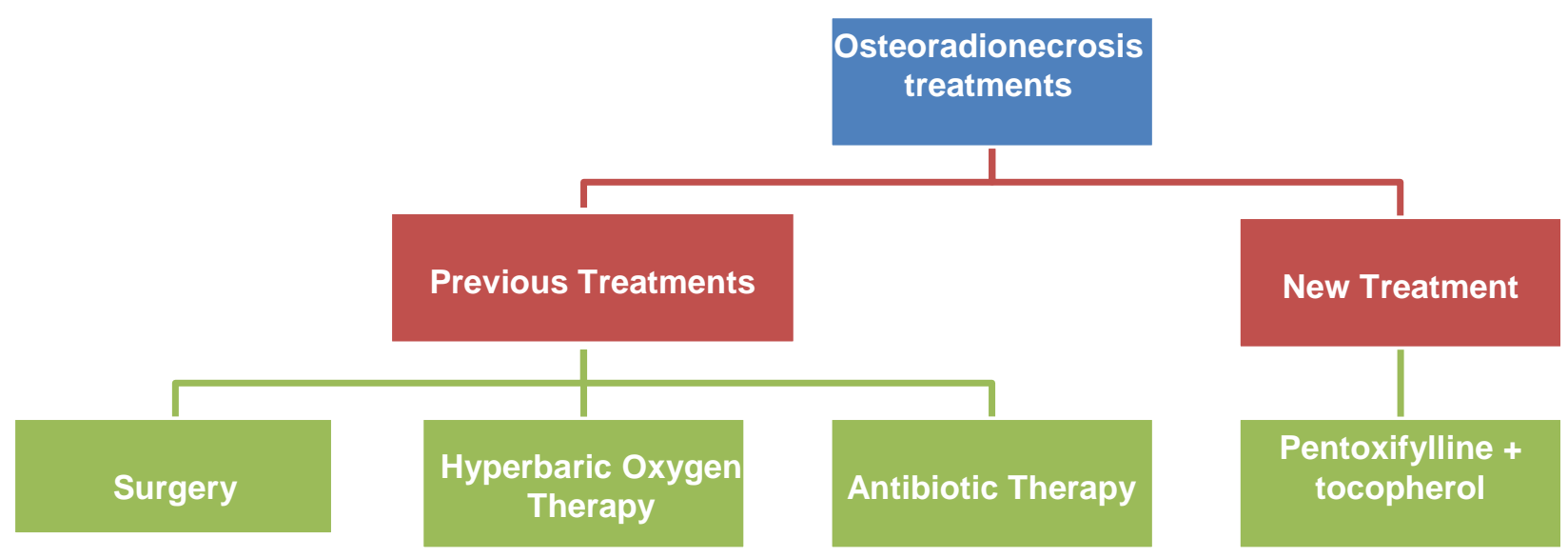

Adapted from Fan et al., (2014).

\section{Pentoxifylline and tocopherol}

Pentoxifylline is a tri-substituted methylxanthine derivative that acts on blood viscosity. This type of drug has an antitumor factor that increases the flexibility of erythrocytes, causes peripheral vasodilation, inhibits inflammatory reactions, inhibits the proliferation of human dermal fibroblasts, the production of extracellular matrix and increases collagenase activity, causing an improvement in blood flow and decreasing its viscosity, consequently increasing the flow and oxygenation in the microcirculation [17].

The exact mode of action that leads to clinical improvement with the use of pentoxifylline is not well defined, however, good results have been observed in studies with animals and patients with peripheral arterial disease, 
such as intermittent claudication or characteristic symptoms of circulatory failure lower limbs [11].

Pentoxifylline improves peripheral blood flow, reduces blood viscosity, improves microcirculation, and increases tissue oxygenation. In addition, pentoxifylline has an antitumor necrosis factor alpha (anti-TNF$\alpha)$ effect, inhibiting dermal fibroblasts, increasing collagenase activity. Decreased TNF- $\alpha$ levels induce a reduction in interleukin (IL) production, this was observed among patients with acute coronary syndromes treated with pentoxifylline compared to placebo. Pentoxifylline also reduces the synthesis of pro-inflammatory cytokines, including TNF- $\alpha$ in recurrent aphthous lesions. Furthermore, a decrease in the duration of soft tissue necrosis has been reported when associated with radiation [24].

Another class of drugs that has been studied are Tocopherols, which are organic chemical compounds consisting of various methylated phenols. These drugs are fat-soluble antioxidants that act by scavenging reactive oxygen species generated during oxidative stress produced by endogenous enzymes, protecting cell membranes against lipid peroxidation [11].

Tocopherol's mechanisms of action can reduce inflammation and stimulate healing, it reduces tissue fibrosis and is a potent scavenger of free radicals, which can reduce damage in tissue necrosis. Tocopherol improves endothelial function in patients with hypercholesterolemia or advanced atherosclerosis [25].

Tocopherol E is a powerful antioxidant agent that protects the phospholipid membrane from oxidative damage by scavenging reactive oxygen species generated during oxidative stress, this protects the cell membrane against lipid peroxidation. It also increases the relative concentration of antioxidants in the zone subjected to radiation and helps to reduce reactive oxygen species, promoting normal healing of damaged endothelial tissue [26].

The treatment of patients with Pentoxifylline, Tocopherol and Clodronate-PENTOCLO combined, proved to be effective in septic reduction through the chronification of the ORN, and still showing that the three drugs are available at a low cost, being tolerated by the body and bringing benefits to the patient. It should be remembered that much remains to be investigated about the exact mechanisms of action of these drugs [17].

Clodronate is a new generation of bisphosphonate that inhibits bone 
resorption, reducing the number and activity of osteoclasts, unlike previous bisphosphonates, which act directly on osteoblastic cells by increasing bone formation and reducing fibroblast proliferation [27].

Epstein et al. [28] studied the use of pentoxifylline and tocopherol in six patients with bisphosphonate-associated osteonecrosis. Patients were followed up for an average of 10 months, with a $74 \%$ decrease in bone exposure area, considering that this therapy may represent a therapeutic alternative to control bisphosphonate-associated osteonecrosis.

Magremanne and Reychler [29] presented a case of osteonecrosis developing after three annual zoledronic acid infusions for the treatment of corticosteroid-induced osteoporosis. The proposed treatment was pentoxifylline $400 \mathrm{mg}$ twice a day and tocopherol 1,000 IU single dose for 12 months, with monthly follow-up. The situation was successfully managed through conservative treatment.

Rice et al. [30] performed a literature review dividing the forms of treatment for ORN into two groups: conservative treatment and surgical treatment; highlighting the use of antifibrotic drugs (pentoxifilline, tocopherol and clodronate). They realized that over the years a new understanding of the pathophysiology of ORN has led to the development of new therapeutic approaches. However, in advanced cases of ORN, surgical treatment with microvascular grafting and reconstructive techniques for bone and soft tissue remains the most reliable option.

Robard et al. [31] observed that the pentoxifylline - tocopherol combination decreases radiotherapyinduced superficial fibrosis. They evaluated, through a retrospective study, 27 patients with a mean age of 65 \pm 12 years, presented with ORN in the mandible induced by radiotherapy in the head and neck region and treated by the PENTOCLO protocol, between January 2010 and March 2011.

$$
\text { Clinical evaluation }
$$

was performed by measuring mucosal ulceration and radiological examination, before and after treatment. An improvement in mucosal ulceration was seen in 16 of 21 patients (80\%) after 3 months, and 12 of 17 patients (75\%) after 6 months of PENTOCHL.

Clinical cure was achieved in 16 patients (80\%). Radiological cure was achieved after 3 months in 9 of 20 patients (49\%). The safety and efficacy of the treatment were evaluated through clinical intraoral examination, and assessment of feeding, weight and analgesic consumption. 
Use of Pentoxypline and Tocopherol in the treatment of Osteorradionecrosis: a literature review

The PENTOCLO protocol achieved clinical and radiological regression of ORN with reduced indications for major surgery. These preliminary results must be confirmed through studies that comprise quality of life assessment. D'Souza et al. [32], through a retrospective analysis of the treatments of osteoradionecrosis (ORN) of the mandible using pentoxifylline, tocopherol, and doxycycline, evaluated 71 patients treated for ORN of the mandible, during a period of 15 years until January of 2011; were classified using the Notani classification: grade I $(\mathrm{n}=28)$, grade II $(\mathrm{n}=16)$, and grade III $(\mathrm{n}=27)$.

Twelve patients with grade I ORN, 3 with grade II, and 10 with grade III, were prescribed drug treatment. Of these, three with grade I, and two with grade II ORN were cured; disease progression had stopped and there was satisfactory symptom control in eight grade I and four grade III. Patients who did not respond to conservative treatment were evaluated for the need for free flap reconstruction. Of the 39 patients diagnosed before the introduction of the drug protocol, 20 (51\%) required resection and reconstruction with a free graft compared with only 8 out of 32 patients (25\%) after drug therapy was instituted by the team.

\section{Discussion and Conclusion}

Despite technological advances in radiotherapy devices and the improvement of surgical techniques, the incidence of osteoradionecrosis (ORN) has not decreased in recent decades. Depending on the institution analyzed, the incidence of ORN has varied from $1 \%$ to $40 \%$ of cases [12].

The most observed adverse effects of head and neck radiotherapy in the literature were: mucositis, hyposalivation, radiation caries lesions, dysphagia, trismus, tissue necrosis and osteoradionecrosis. Oral health care should be part of the multidisciplinary care of cancer patients in order to provide a comprehensive concomitant treatment [4, 14-16].

There was a consensus in the studies that prevention is the main factor in the management of the irradiated patient. The evaluation of dental and periodontal status are very important to define the preradiotherapy approach $[4-5,18]$.

During the last 80 years a series of theories about the pathogenesis of ORN have been proposed, with consequent implications for its treatment. Until the tissue hypoxia theory and its consequences were accepted as the primary cause, and this led to the use of HBOT, both for the treatment and prevention of 
complications of radiotherapy in the head and neck $[13,18,26]$.

ORN in the early stages can be managed conservatively, using a combination of treatment options. In advanced cases of ORN (pathological fracture, orocutaneous fistula), surgical treatment remains the only treatment option available. A new understanding of the pathophysiology of ORN has led to the development of new therapeutic management regimens [30].

Recent discussions regarding the pathogenesis of ORN have proposed that bone damage is caused by radiation-induced fibrosis. Bone tissue cells are damaged as a result of acute inflammation, free radicals, and chronic activation of fibroblasts by a variety of growth factors. Thus, new treatments with promising results have been developed, including the use of pentoxifylline, a peripheral vasodilator that also inhibits fibrosis, and tocopherol to reduce the damage caused by free radicals [17-18, 26, 33].

Epstein et al. [28] evaluated six patients with bisphosphonate-associated osteonecrosis, establishing a combination of pentoxifylline, tocopherol and chlorhexidine as a therapy and observed the evolution of the area of bone exposure of these patients for 10 months and found that there was a decrease of $74 \%$ of exposure. The authors demonstrated that therapy with pentoxifylline and tocopherol may represent a therapeutic alternative to control bisphosphonateassociated osteonecrosis. However, they believe that more studies are needed to confirm this therapy.

Delanian et al. [27] adopted an alternative strategy for the treatment of mandible ORN, based on this new concept of ORN pathophysiology and fibro-atrophic bone etiology. Thus, they evaluated the combination of pentoxifylline, tocopherol and clodronate-PENTOCOLUS in patients with refractory ORN. There was a reduction in bone exposure and mucosal healing after six months in most patients. It was also found that longterm treatment with PENTOCOL was effective, safe and managed to treat refractory ORN, inducing mucosal and bone healing with improvement of symptoms after 9 months of follow-up. Randomized studies are needed to confirm the conclusion.

Patel et al. [33] retrospectively analyzed data on 390 tooth extractions in 82 patients who had undergone radiation therapy for head and neck cancer. In one group of patients, pentoxifylline and tocopherol were used prophylactically to reduce the risk of ORN after tooth extractions. Only one patient $(1.2 \%)$ developed ORN (rate / tooth $0.26 \%$ ). Medications were 
administered approximately 11 weeks before surgery and for 13 weeks postoperatively. The incidence they found was lower than that normally associated with tooth extractions in irradiated patients, which is $7 \%$ [4].

Tocopherols are fat-soluble antioxidants, but they also appear to have many other functions in the body. One of the functions of endogenous tocopherol is to eliminate reactive oxygen species generated during oxidative stress that escape antioxidant activity in vivo enzymes, to protect cell membranes against lipid peroxidation, and, in part, to inhibit TGF- $\beta 1$ and pro collagen gene expression [17-18].

It has been seen that the combination therapy of PentoxifyllineTocopherol proved to be effective in the chronic progressive septic reduction of the ORN of the mandible, being a complementary alternative to the existing therapies in the treatment of osteoradionecrosis. These two drugs act synergistically, have potent antifibrotic agents, are well tolerated in the body, and are available at a low cost. When associated, they reduce fibrosis, capturing oxygen species, protecting the cell membrane against lipid peroxidation, and partially inhibiting TGF- $\beta$ and pro collagen gene expression $[11,17-18,26]$.
McLeod et al. [11] analyzed a series of 12 patients with ORN who had used pentoxifylline and tocopherol. One patient had to stop treatment because of the side effects of pentoxifylline; three reported difficulty in swallowing the tablet. Five patients improved their condition, in five there was no progression of the disease and in two cases the lesion progressed. The authors considered that the use of pentoxifylline and tocopherol in the treatment of patients with maxillary ORN could not be supported based on the results found in this series, but prospective data acquisition with a larger group would be prudent before such a promising treatment is abandoned [11].

Patel et al. [33] performed a retrospective analysis of 62 patients with ORN, evaluating the potential of treatment with pentoxifylline and tocopherol. In the group that used only pentoxifylline and tocopherol, 56\% showed an improvement, however, paradoxically, when it was combined with antibiotics, only $27 \%$ of the cases resolved. The authors believed that ideally they would need to carry out a randomized clinical trial comparing treatment with pentoxifylline and tocopherol, antibiotic therapy and HBOT.

Bohn et al. [34] reported treatment success with this prescription protocol in three cases. They underwent 
radiotherapy to treat malignant tumors in the head and neck region and developed ORN. All patients achieved complete remission in less than one year, with complete healing of bone exposure and no clinical symptoms. Showing that the combination of pentoxifylline and tocopherol can be beneficial in cases of radiation-induced bone necrosis, avoiding more aggressive treatments and reducing morbidity [34].

Hayashi et al. [25] followed 13 patients who presented to the service with exposed bone after radiation doses for head and neck cancer. Patients were treated with pentoxifylline 400mg twice daily and tocopherol 1,000 IU single dose. Eleven of 13 patients showed improvement and resolution of the exposed bone. The authors suggested that this treatment modality for ORN appears safe and effective [25].

Robard et al. [31] suggested that PENTOCLO treatment appears to be effective, low-cost, and almost devoid of adverse effects. It is, therefore, a good alternative, among the available treatments, for the management of mandibular ORN. The PENTOCLO protocol achieved clinical and radiological regression of ORN with a reduction in indications for major surgeries. These preliminary results must be confirmed through placebocontrolled, prospective and randomized studies [31].
D'Souza et al. [32] performed a retrospective analysis of patients with ORN of the mandible treated with pentoxifylline, tocopherol and doxycycline. Of 39 patients diagnosed with ORN who did not use the drug protocol, 20 patients $(51 \%)$ required resection and reconstruction with a free graft, compared with only 8 patients out of $32(25 \%)$, of whom drug therapy was instituted [32].

From the researched literature on this subject and based on the current understanding of ORN, it was noted that there are several effective therapeutic strategies to improve tissue healing, but a "gold standard" treatment has not yet been established. Optimal treatment may involve the combination of antifibrotic drugs, antibiotics, HBOT and sequestrectomy as a final treatment option [32].

The authors' expectations are that, in the near future, surgery may be restricted to severe cases (ie, with pathological fracture dislocation and severe bone exposure). More prospective randomized controlled studies are needed to establish a new therapeutic protocol $[8,11,17,30,22]$.

\section{References}

[1] Instituto Nacional de Câncer. Estimativa 2016: incidência de câncer no Brasil / Instituto Nacional de Câncer 
José Alencar Gomes da Silva - Rio de Janeiro: INCA, 2015.

[2] Rolim AEH, Da Costa LJ, Ramalho LMP. Repercussões da radioterapia na região orofacial e seu tratamento. Radiologia Brasileira. 2011, 44(6):388-395.

[3] Kuhnt T, Stang A, Wienke A, Vordermark D, Schweyen R, Hey J. Potential risk factors for jaw osteoradionecrosis after radiotherapy for head and neck cancer. Radiat Oncol. 2016 Jul 30;11:101. doi: 10.1186/s13014016-0679-6.

[4] Nabil S, Samman N. Incidence and prevention of osteoradionecrosis after dental extraction in irradiated patients: a systematic review. Int J Oral Maxillofac Surg. 2011 Mar;40(3):229-43. doi: 10.1016/j.ijom.2010.10.005.

[5] Almeida FCS, Cazal C, Araujo ME, Brandão T, Vaccarezza GF, Silva DP. Tratamento conservador de osteorradionecrose de mandíbula: equidade e racionalização de recursosrelato de casos. Revista Brasileira de Ciências da Saúde. 2012, 16(1):63-70.

[6] Marx RE. Osteoradionecrosis: a new concept of its pathophysiology. J Oral Maxillofac Surg. 1983 May;41(5):283-8. doi: 10.1016/0278-2391(83)90294-x.

[7] Chrcanovic BR, Reher P, Sousa AA, Harris M. Osteoradionecrosis of the jaws--a current overview--part 1 : Physiopathology and risk and predisposing factors. Oral Maxillofac
Surg. $\quad 2010 \quad$ Mar;14(1):3-16. doi: 10.1007/s10006-009-0198-9.

[8] Grimaldi N, Sarmento V, Provedel L, Almeida D, Cunha S. Conduta do cirurgião-dentista na prevenção e tratamento da osteorradionecrose: revisão de literatura. Rev Bras Cancerol. 2005, 51(4):319-24.

[9] Monteiro L, Barreira E, Medeiros L. Osteorradionecrose dos maxilares. Rev Port Estomatol Cir Maxilofac. 2005, 46:49-62.

[10] Batinjan G, Filipovic Zore I, Vuletic M, Rupic I. The use of ozone in the prevention of osteoradionecrosis of the jaw. Saudi Med J. 2014 Oct;35(10):12603.

[11] McLeod NM, Pratt CA, Mellor TK, Brennan PA. Pentoxifylline and tocopherol in the management of patients with osteoradionecrosis, the Portsmouth experience. $\mathrm{Br} \mathrm{J}$ Oral Maxillofac Surg. 2012 Jan;50(1):41-4. doi: 10.1016/j.bjoms.2010.11.017.

[12] Curi MM, Kowaslki LP. Osteorradionecrose de mandíbula e maxila. Revista Brasileira de Cirurgia de cabeça e pescoço. 2003, 31:49-53, 2003.

[13] Aldunate JLCB, Coltro PS, Busnardo FF, Ferreira MC. Osteorradionecrose em face: fisiopatologia, diagnóstico e tratamento. Rev. Bras. Cir. Plást.2010;25(2):381-387.

[14] Huber MA, Terezhalmy GT. The head and neck radiation oncology 
patient. Quintessence Int. 2003 Oct;34(9):693-717.

[15] Bonan PRF, Lopes MA, Pires FR, Almeida OP. Dental management of low socioeconomic level patients before radiotherapy of the head and neck with special emphasis on the prevention of osteoradionecrosis. Brazilian dental journal. 2006, 17(4):336-342.

[16] Vissink A, Jansma J, Spijkervet FK, Burlage FR, Coppes RP. Oral sequelae of head and neck radiotherapy. Crit Rev Oral Biol Med. 2003;14(3):199-212. doi: 10.1177/154411130301400305.

[17] Fan H, Kim SM, Cho YJ, Eo MY, Lee SK, Woo KM. New approach for the treatment of osteoradionecrosis with pentoxifylline and tocopherol. Biomater Res. $\quad 2014 \quad$ Sep 29;18:13. doi: 10.1186/2055-7124-18-13.

[18] Lyons A, Ghazali N. Osteoradionecrosis of the jaws: current understanding of its pathophysiology and treatment. Br J Oral Maxillofac Surg. 2008 Dec;46(8):653-60. doi: 10.1016/j.bjoms.2008.04.006.

[19] Notani K, Yamazaki Y, Kitada H, Sakakibara N, Fukuda H, Omori K, Nakamura M. Management of mandibular osteoradionecrosis corresponding to the severity of osteoradionecrosis and the method of radiotherapy. Head Neck. 2003 Mar;25(3):181-6. doi: 10.1002/hed.10171.

[20] Støre G, Boysen M. Mandibular osteoradionecrosis: clinical behaviour and diagnostic aspects. Clin Otolaryngol
Allied Sci. 2000 Oct;25(5):378-84. doi: 10.1046/j.1365-2273.2000.00367.x.

[21] Epstein J, van der Meij E, McKenzie M, Wong F, Lepawsky M, StevensonMoore P. Postradiation osteonecrosis of the mandible: a long-term follow-up study. Oral Surg Oral Med Oral Pathol Oral Radiol Endod. 1997 Jun;83(6):65762. doi: 10.1016/s1079-2104(97)90314-0.

[22] Costa DA, Costa TP, Netto EC, Joaquim N, Ventura I, Pratas AC, Winckler P, Silva IP, Pinho AC, Sargento IG, Guerreiro FG, Moreira AR. New perspectives on the conservative management of osteoradionecrosis of the mandible: A literature review. Head Neck. 2016 Nov;38(11):1708-1716. doi: 10.1002/hed.24495.

[23] Bui QC, Lieber M, Withers HR, Corson K, van Rijnsoever M, Elsaleh $\mathrm{H}$. The efficacy of hyperbaric oxygen therapy in the treatment of radiationinduced late side effects. Int J Radiat Oncol Biol Phys. 2004 Nov 1;60(3):871-8. doi: 10.1016/j.ijrobp.2004.04.019.

[24] Delanian S, Depondt J, Lefaix JL. Major healing of refractory mandible osteoradionecrosis after treatment combining pentoxifylline and tocopherol: a phase II trial. Head Neck. 2005 Feb;27(2):114-23. doi: 10.1002/hed.20121.

[25] Hayashi M, Pellecer M, Chung E, Sung E. The efficacy of pentoxifylline/tocopherol combination in the treatment of osteoradionecrosis. Spec Care Dentist. 2015 NovDec;35(6):268-71. doi: 10.1111/scd.12124. 
[26] Delanian S, Porcher R, Balla-Mekias S, Lefaix JL. Randomized, placebocontrolled trial of combined pentoxifylline and tocopherol for regression of superficial radiationinduced fibrosis. J Clin Oncol. 2003 Jul 1;21(13):2545-50. doi: 10.1200/JCO.2003.06.064.

[27] Delanian S, Chatel C, Porcher R, Depondt J, Lefaix JL. Complete restoration of refractory mandibular osteoradionecrosis by prolonged treatment with a pentoxifyllinetocopherol-clodronate combination (PENTOCLO): a phase II trial. Int J Radiat Oncol Biol Phys. 2011 Jul 1;80(3):832-9. doi: 10.1016/j.ijrobp.2010.03.029.

[28] Epstein MS, Wicknick FW, Epstein JB, Berenson JR, Gorsky M. Management of bisphosphonateassociated osteonecrosis: pentoxifylline and tocopherol in addition to antimicrobial therapy. An initial case series. Oral Surg Oral Med Oral Pathol Oral Radiol Endod. 2010 Nov;110(5):593-6. doi: 10.1016/j.tripleo.2010.05.067.

[29] Magremanne $M$, Reychler $\mathrm{H}$. Pentoxifylline and tocopherol in the treatment of yearly zoledronic acidrelated osteonecrosis of the jaw in a corticosteroid-induced osteoporosis. J Oral Maxillofac Surg. 2014 Feb;72(2):334-7. doi: 10.1016/j.joms.2013.06.188.

[30] Rice N, Polyzois I, Ekanayake K, Omer O, Stassen LF. The management of osteoradionecrosis of the jaws--a review. Surgeon. 2015 Apr;13(2):101-9. doi: 10.1016/j.surge.2014.07.003.

[31] Robard L, Louis MY, Blanchard D, Babin E, Delanian S. Medical treatment of osteoradionecrosis of the mandible by PENTOCLO: preliminary results. Eur Ann Otorhinolaryngol Head Neck Dis. 2014 Dec;131(6):333-338. doi: 10.1016/j.anorl.2013.11.006.

[32] D'Souza J, Lowe D, Rogers SN. Changing trends and the role of medical management on the outcome of patients treated for osteoradionecrosis of the mandible: experience from a regional head and neck unit. Br J Oral Maxillofac Surg. 2014 Apr;52(4):356-62. doi: 10.1016/j.bjoms.2014.01.003.

[33] Patel V, Gadiwalla Y, Sassoon I, Sproat C, Kwok J, McGurk M. Prophylactic use of pentoxifylline and tocopherol in patients who require dental extractions after radiotherapy for cancer of the head and neck. Br J Oral Maxillofac Surg. 2016 Jun;54(5):547-50. doi: 10.1016/j.bjoms.2016.02.024.

[34] Bohn JC, Schussel JL, Stramandinoli-Zanicotti RT, Sassi LM. Tissue repair in osteoradionecrosis using pentoxifylline and tocopherol-report of three cases. Oral Maxillofac Surg. 2016 Mar;20(1):97-101. doi: 10.1007/s10006-015-0522-5.

Conflict of interest: The author declares no conflicts of interest.

Acknowledgements: None.

Funding: None. 
How to cite this article: Gusmão JNFM,

Alves RL, Santos ES. Use of Pentoxypline and Tocopherol in the treatment of Osteorradionecrosis: a literature review. Brazilian Journal of Case Reports. 2021 Abr-Jun; 01(2):59-77. 